\title{
The remote and high precision current control system of multiple high power LEDS
}

\author{
Yi-Huang Lin, Juan Liu, Ling-Ling Sun, Xi Chen, Cun-Kai Qiu and Gao-Feng Zheng* \\ Department of Instrument and Electrical Engineering, Xiamen University, Xiamen \\ 361005, China \\ E-mail: zheng_gf@xmu.edu.cn \\ *Corresponding author
}

\begin{abstract}
For the remote control of multi-channel high power LEDS, a high-precision LED curren control system is proposed. A microcontroller system based on STM32F217ZET containing a four-channel high power LED driver circuit based on LT3755-1 was designed. The high-precision current control compensation algorithm is presented, which divides the current control function into three parts and adds proportionality and shift coefficients to the function, improving the current control accuracy from $25 \%$ to $\pm 1 \%$. The output current of each channel can be continuously adjusted from $200 \mathrm{~mA}$ to $1800 \mathrm{~mA}$, with the single channel output power able to reach $90 \mathrm{~W}$ and the output voltage able to reach $50 \mathrm{~V}$. The system proposed can be monitored via TCP/IP through the remote PC interface.
\end{abstract}

Keywords: LED Constant Current Drive Circuit; High Precision; Control Algorithm; Remote Control.

\section{Introduction}

With the advantages of small size [1], long life, high efficiency [2] and easily installing, high-power LED has been widely used in automotive areas, medical areas, display areas and lighting areas [3].

LED is a kind of electroluminescent device, and the impact that current has on its luminescent properties attracted wide attention of researchers. Studies have shown that there is a close link between the current through the LED and the characteristic parameters of LED, such as luminous flux [4], the main wavelength [5], color temperature, luminous efficiency [6] and chroma [7], etc. Precision current regulation is the key to studying the LED characteristic parameters, thus the LED drive circuit design has become the focus of research. Constant current power supply is generally used to drive the high-power LED, which ensures the stability of brightness [8]. With the advantages of short development cycle and simple design, the mature LED driver chip has become 
the main choice for the scholar to design the constant current source [9-12]. However, the non-linear characteristic of the LED voltage-current, the nonlinear characteristic of the LED driver chip system, and the sampling resistor affected by temperature will cause the current control accuracy of the control system to be low, which affects the research of LED characteristic parameter and the quality of photo image [4 -7].

In addition, with the development of the Internet system, the remote intelligent health monitoring, the remote lighting control and the remote fault diagnosis of the high- power LED will be able to gradually improve [13].

Therefore, using the LED driver chip to achieve high-precision current control has become a technical difficulty. This paper introduces a high-precision current control system based on STM32F217ZET containing a four-channel high power LED driver circuits based on LT3755-1. Through high-precision current compensation algorithm, which divides the current control function into 3 parts and adds proportionality and shift coefficients to the function, the current control system achieves four-channel LED current high-precision regulation, with control accuracy up to $\pm 1 \%$, and has a remote control function.

\section{Design of Multi-channel LED Current Control System}

\subsection{LED voltage-current characteristics}

LED consists of a PN junction with a unidirectional continuity. The applied forward voltage $V_{L E D}$ makes the forward current $I_{A}$ large enough to emit LED. The greater $I_{A}$ is, the stronger the luminous intensity is. And different colors of

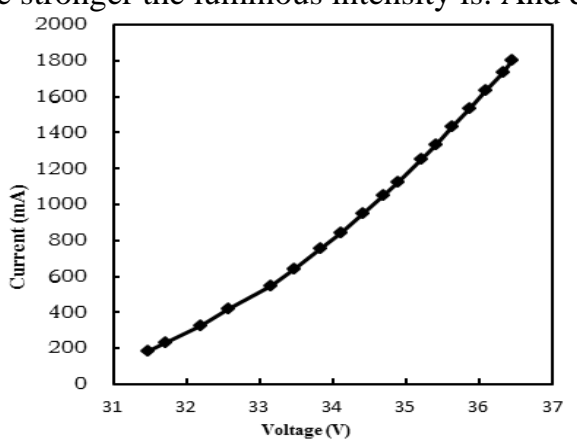

Fig. 1 The voltage-current characteristics curve of 22 5W LEDs in parallel

LED lights have different threshold voltages. Figure 1 shows the voltagecurrent characteristics curve of $225 \mathrm{~W}$ LEDs which have two groups in parallel and each group has 11 LEDS in series. As shown from the Fig. 1, only when the 
forward voltage $V_{L E D}$ is greater than the threshold voltage can the forward current $I_{A}$ increase from zero with increasing exponentially. The change in $I_{A}$ is great whilethe change in $V_{L E D}$ is small. Thus, the voltage-current characteristics curve of the LED is similar to the PN, having an exponential characteristic. The $I_{A}$ changes from 200 to $1800 \mathrm{~mA}$, while the change of $V_{L E D}$ is generally between 31-37V.

\subsection{System components}

Fig. 2 shows the component of multi-channel LED current control system, including PC control surface, communication module, STM32F217ZET SCM minimum system, EEPROM memory module, DAC6574 module, temperature detection module and LT3755-1 LED constant current controller.

A minimum control system based on microcomputer STM32F217ZET produced by stmicroelectronics company was created. The system adoptes analog dimming of LT3755-1, which can change the LED current through the change of analog voltage to the chip. When $I_{A}$ is set in the PC control interface, the SCM calculates the analog voltage needed to control the $I_{A}$ with high precision compensation algorithm. The SCM controls the DAC6574 module through $\mathrm{I}^{2} \mathrm{C}$ communication, and then the DAC6574 sends the high precision control voltage to the LT3755-1 LED constant current controller to control the current of the LED. Temperature detection module can monitor the temperature of the LED. When the temperature is over the set temperature, the alarm signal is given and cooling fan will be started. The EEPROM memory module stores last working parameters of each LED, the parameters used in the compensation algorithm and system setting temperature.

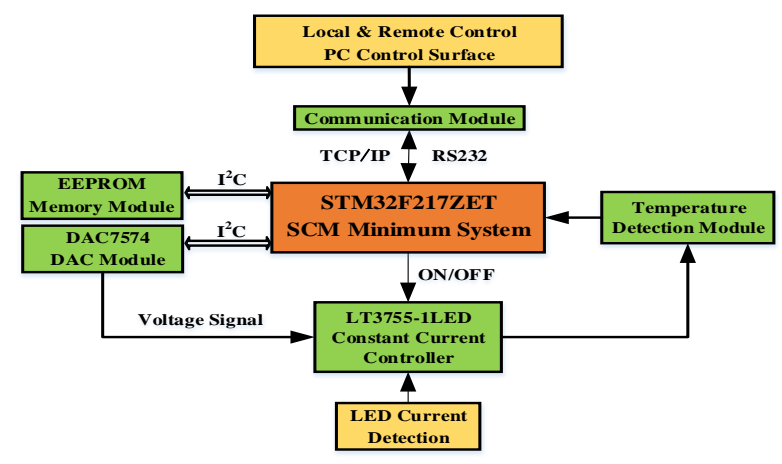

Fig. 2 The diagram of multi-channel LEDs current control system 
The SCM selected comes with an Ethernet module. And an external PHY chip ENC28J60 is added to enable Ethernet communications, which allows the data transfer rate of 10/100 Mbit/s. The TCP/IP parameters are set in the PC control interface, which can control the LED on a local Ethernet through TCP/IP protocol stack, increasing the communication distance, improving the data transmission speed, ensuring the stability of data transmission. As shown in Fig. 3, connecting the local Ethernet to the Internet [14], we can set the LED parameters in the PC interface to control the LED current from a remote distance through the Internet and monitor the working state of LED. When the LED breaks down, the system automatically alarms and fault information is given, which is convenient for the operation personnel to maintain the LED.

\subsection{The PC control interface}

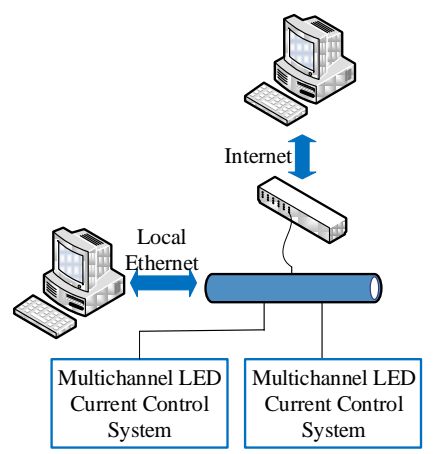

Fig. 3 The remote control diagram based on the Ethernet

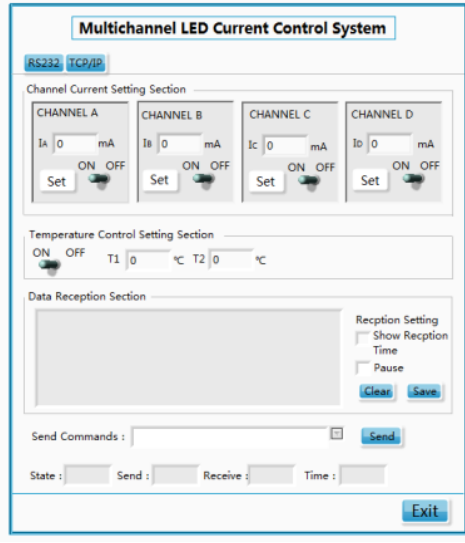

Fig. 4 The PC control interface

The PC control interface of the system is shown in Fig. 4. We can set the working state and the current value of each channel, and send commands or receive data in this interface.

\subsection{Design of LED constant current controller}

The large current DC-DC converter LT3755-1 can realize the control of constant current, which satisfies the requirement of stability of light source [15]. There is a current closed-loop control inside the circuit which improves the efficiency, reduces the size of the external devices and reduces the hardware cost. However, using the analog dimming control strategy will lead to the problem of the low current control accuracy. 
The schematic diagram of the constant source controller whose core is the Boost circuit is shown in Fig. 5. When the circuit is in stable state, the switch $Q 1$ is on, then the power supply $V C C$ charges the inductor $L 6$ and the inductor current rises with a certain speed, then a certain amount of energy is stored in the inductor, meantime C6-C8 power the LED. When $Q 1$ is off, since the inductor current cannot change suddenly, the $V C C$ and $L 6$ charge C6-C8 and provide energy to the LED. Thus, at the end of a switching cycle, the output voltage of capacitor has been greater than the VCC. LT3755-1 controller detects the voltage between ISP and ISN to realize constant current driver. The SCM control the PWMOUT pin to control LED on or off. That is, the SCM can control LED by changing level of the PWM pin.

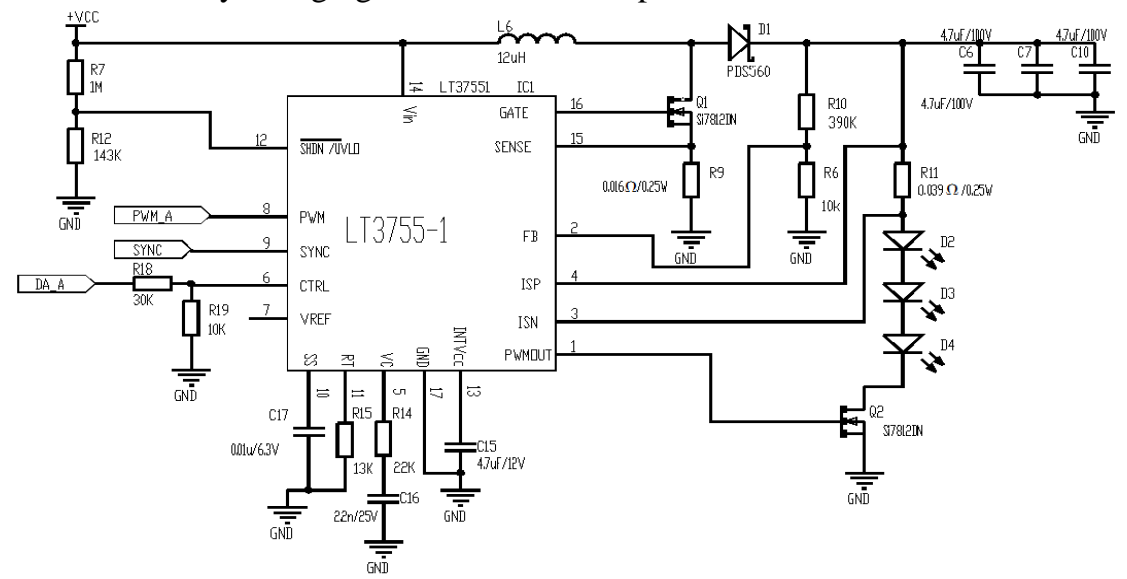

Fig. 5 The schematic diagram of the constant source controller based on LT3755-1

The current of LED can be changed by setting the CTRL pin voltage of LT3755-1. When the $V_{C T R L}$ is less than $1.1 \mathrm{~V}$, the current of LED $I_{L E D}$ is set as follows:

$$
I_{L E D}=\frac{V_{C T R L}-100 \mathrm{mV}}{10 R_{L E D}}
$$

Where $R_{L E D}$ is the LED sampling resistor, seen as $R_{11}$ in Fig. 5.

The system adopts DAC7574 to offer variable voltage for CTRL pin to control the LED current. This is a low power consumption, 10-bit accuracy, $\mathrm{I}^{2} \mathrm{C}$ communication, and 4-channel DAC. The relationship between output voltage of each channel and the digital D given by the SCM is as follows:

$$
V=\frac{D}{1024} \times 5
$$

The maximum voltage of DAC7574 is $5 \mathrm{~V}$, so to meet the design requirements voltage giving to CTRL pin was divided through $R_{18}$ and $R_{19}$. 
The maximum output current of LED is 1.8A. Considering the margin and sampling resistance selection, $R_{L E D}=0.039 \Omega$ was finally chosen. The maximum output voltage of LED is $50 \mathrm{~V}$, the maximum output voltage $V_{\text {out }}$ is set as follows:

$$
V_{\text {out }}=1.25 \frac{R_{10}+R_{6}}{R_{6}}
$$

So $R_{10}=390 \mathrm{~K}, R_{6}=10 \mathrm{~K}$ were chosen.

The soft start time of LT3755-1 can be determined by the value of $C_{17}$, as shown in Eq. (4).We set the soft start time $2 \mathrm{~ms}$, thus $C_{17}=0.01 \mathrm{uF}$.

$$
t=\frac{C_{X} 2 \mathrm{~V}}{10 \mu \mathrm{A}}
$$

The switching frequency of $Q 1$ can be programmable between $100 \mathrm{KHz}$ to $1 \mathrm{MHz}$ by changing the $R_{15} . R_{15}=13 \mathrm{~K} \Omega$ was selected, then fosc $=800 \mathrm{KHz}$. For the booster topology, $R_{S E N C E \text {, Boost }}$ and $L_{B o o s t}$ need to satisfy the Eq. (5) and Eq. (6), shown as $R_{9}$ and $L_{6}$ in Fig. 6.

$$
\begin{gathered}
R_{\text {SENCE,Boost }} \leq \frac{V_{I N} \bullet 0.07 \mathrm{~V}}{V_{L E D} \bullet I_{L E D}} \\
L_{\text {Boost }}=\frac{T_{S W} \bullet R_{S E N C E} \bullet V_{I N}\left(V_{L E D}-V_{I N}\right)}{V_{L E D} \bullet 0.02 \mathrm{~V}}
\end{gathered}
$$

Where $V_{I N}$ is the input voltage $V C C, V_{L E D}$ is the maximum voltage of the LED, $I_{L E D}$ is the maximum current through the LED, and $T_{S W}$ is switch control cycle of $Q 1$. Then $R_{S E N C E}$ was $16 \mathrm{~m} \Omega$ and $L_{\text {Boost }}$ was $12 \mathrm{uH}$.

\subsection{High precision current control compensation algorithm}

From Eq. (1) and Eq. (2), we can obtain Eq. (7) and Eq. (8). We can infer that $R_{L E D}$ has a great influence on the control precision of the current control system, and the minimum control current is $0.03 \mathrm{~mA}$ theoretically.

However, when the system is on operation, the non-linear characteristic of the LED voltage-current and LED driver chip system, and the sampling resistor affected by temperature will affect the current control accuracy which means the control function of LED current can not be same as Eq. (7). The whole system is nonlinear.

$$
\begin{gathered}
D=\frac{4096 R_{L E D} \bullet I_{L E D}+409.6}{5} \\
\frac{d I_{L E D}}{d D}=\frac{5}{4096 R_{L E D}}
\end{gathered}
$$

The current control precision curve of four channels using Eq. (7) to control the LED current is shown in Fig. 8. As shown in Fig. 8, the control accuracy is at about $25 \%$. When current value $(200-600 \mathrm{~mA})$ is small, the current control 
accuracy is low. As the current increases, the control accuracy of the current (600-1200mA) has improved to the vicinity of $\pm 2 \%$. With the current continuing to increase, to the high current value(1200-1800mA), the control accuracy of the current is reducing. As shown from the figure, the current control accuracy without compensation algorithm has three distinctive sections, and the overall control accuracy is low.

In order to improve the current control precision of the system, we adopt high precision compensation current control algorithm according to the Fig. 8. We divide Eq. (7) into three parts according to the LED current, and add proportionality coefficient $p$ and shift coefficient $s$ into every control function of each channel. The current was divided into three parts: 200 to $600 \mathrm{~mA}, 600-$ $1200 \mathrm{~mA}$ and1200-1800mA. Each part was added coefficients $p$ and $s$ respectively. Thus, the final function is as follows:

$$
D=p x \frac{4096 R_{L E D} \bullet I_{L E D}+409.6}{5}+s
$$

The final coefficients $p$ and $s$ have to be determined by the error between the real measured LED current and the current value given. Finally we chose the best $p$ and $s$ according to the current control accuracy characteristic curve.

\section{Results and Discussion}

A four-channel and adjustable LED constant current control system was designed and constructed. The test platform of LED current control system is shown in Fig. 6. The input power $V C C$ is DC $24 \mathrm{~V}$, and 22 white LED of $5 \mathrm{~W}$ with two groups in parallel were adopted as the test light source. Oscilloscope and multimeter are used to test the output voltage $\left(V_{L E D}\right)$ and current $\left(I_{A}\right)$ of the system.

The waveform of $V_{L E D}$ when $I_{A}=1200 \mathrm{~mA}$ is shown in Fig. 7, $V_{L E D}$ swings between $34.9 \mathrm{~V}-35 \mathrm{~V}$. The current control precision curve of four channels with compensation algorithm is shown in Fig. 9.The compensation coefficients $p$ and $s$ of each channel was shown in table 1 .

As shown from Fig. 9, the current control accuracy of four-channel improved from $25 \%$ to $\pm 1 \%$ after the introduction of compensation algorithm. 


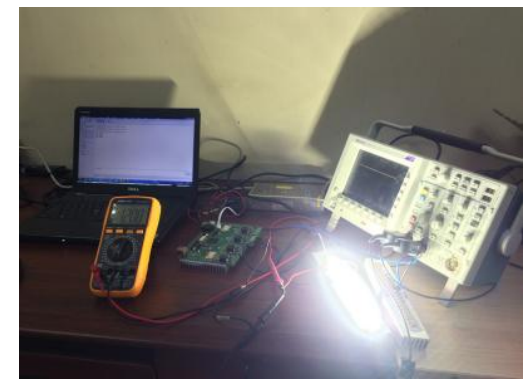

Fig. 6 The test platform of LED current control system $\mathrm{IA}=1200 \mathrm{~mA}$

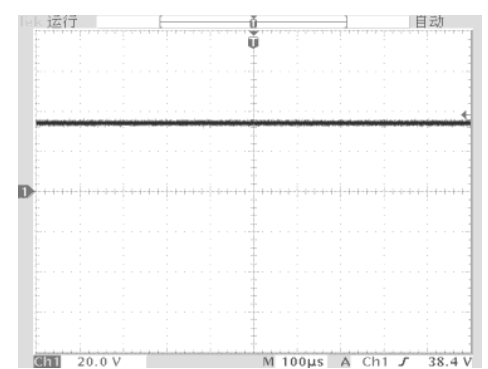

Fig. 7 The VLED waveform when

Improved current control has a distinct feature of three segments like the control function, so there are obvious turning points at the current of $600 \mathrm{~mA}$ and $1200 \mathrm{~mA}$. As shown from Table 1, the $p$ value and $s$ value of each channel is different from each other, for which the system parameter of each channel is different. Each $p$ value and $s$ value shown in Table 1 were got after several attempt and correcting for many times. As shown from Eq. (7), the role of the $p$ value is to suppress the change trend of the control function. When the $D$ value the SCM system gives to the DAC7574 is too large, we can make $p$ less than 1

Tab. 1 The compensation coefficients $\mathrm{p}$ and $\mathrm{s}$ of each channel

\begin{tabular}{ccccc}
\hline $\begin{array}{c}\text { compensation } \\
\text { coefficient }\end{array}$ & $\begin{array}{c}\text { Channel } \\
\text { A }\end{array}$ & $\begin{array}{c}\text { Channel } \\
\text { B }\end{array}$ & $\begin{array}{c}\text { Channel } \\
\text { C }\end{array}$ & $\begin{array}{c}\text { Channel } \\
\text { p1 }\end{array}$ \\
s2 & -10 & 1 & 1.02 & 1.01 \\
p2 & 1 & -13 & -18 & -7 \\
s3 & +1 & 0.99 & 0.98 & 1 \\
p3 & 1 & 0 & 0 & 0 \\
\hline
\end{tabular}

to suppress this change. And when the $p$ value suppress the change trend too much, it is possible to decrease the $D$ value by decreasing the $s$ value, ensuring the overall current control accuracy. Specific values of $p$ and $s$ still need to be determined by field debugging. By debugging 10 sets of multi-channel current control system, we find that when $p$ value is selected between 0.98-1.03, and $s$ value is selected between -18-20, the final current control precision of the whole system will reach $\pm 1 \%$ easily. The current control precision $m$ used in this paper is determined as Eq. (10).

$$
m=\left|\frac{I_{L E D 1}-I_{L E D 2}}{I_{L E D 2}}\right| \times 100 \%
$$

Where $I_{L E D I}$ is the LED current value detected, $I_{L E D 2}$ is the current value given from the $\mathrm{PC}$ interface. 


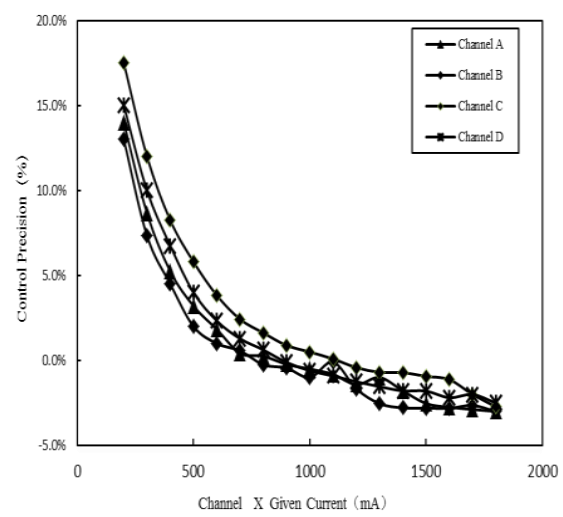

Fig. 8 The current control precision curve of four channels without compensation algorithm

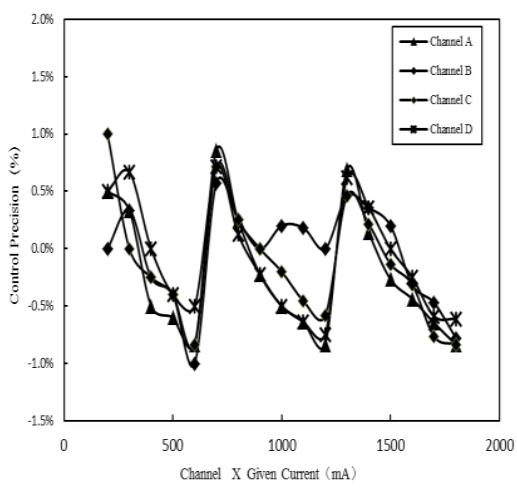

Fig. 9 The current control precision curve of four channels with compensation algorithm

\section{Conclusion}

A set of four-channel LED current control system was designed in this paper, which is pure hardware closed-loop control. It has a fast response to current regulation and circuit structure is simple. The high-precision current control compensation algorithm is presented, which divides the current control function into three parts and adds proportionality and shift coefficients to the function, improving the current control accuracy from $25 \%$ to $\pm 1 \%$. When $\mathrm{p}$ value is selected between $0.98-1.03$, and $\mathrm{s}$ value is selected between $-18-20$, the final current control precision of the whole system will reach $\pm 1 \%$ easily. The output current of each channel can be continuously adjusted from $200 \mathrm{~mA}$ to $1800 \mathrm{~mA}$, with the single channel output power able to reach $90 \mathrm{~W}$ and the output voltage able to reach 50V. The system proposed can be monitored and has the function of fault diagnosis via TCP/IP through the remote PC interface. This system can 
be applied to street lamps and tunnel lights, especially to some high current control precision and remote control applications.

\section{Acknowledgments}

The work was supported by the National Natural Science Foundation of China (Grant nos. 51405408).

\section{References}

1. Yu G. Thermal simulation and optimization design on a high-power LED spot lamp. Optoelectronics Letters, Vol.7(2) (China, 2011), pp. 117-121.

2. Qu Xiaohui. An LED Current Sharing Circuit With High Luminous Efficacy and Individual Dimming. Proceedings of the CSEE, Vol.34(21) (China, 2014), pp.32-40. (In Chinese).

3. Wu F. A precise model of LED lighting and its application in uniform illumination system. Optoelectronics Letters, Vol.7(5) (China, 2014), pp. 334-336.

4. Fang Jinglu. The Influence on Characteristics Parameters for White-LED with Current. Transactions of Electrotechnical Society, Vol.28(2) (China,2013), pp. 234-238. (In Chinese).

5. Li Junfei. The Influence on Optical Parameters for Power White-LED with Current. Light \& Lighting, Vol.33(2) (China,2009), pp. 37-38. (In Chinese).

6. Bi Changdong. Luminous Efficiency about Amount of Phosphor on the High-Power White LED. Journal of Shanxi University of Science\&Technology, Vol.29(2) (China,2011), pp. 56-59. (In Chinese).

7. Pousset N. Impact of current supply on LED colour. Lighting Research and Technology, Vol.42(1) (China,2010), pp. 371-383.

8. Fu Xiansong. Design of Wind-Solar LED Street Lamp's Driver Circuit. Transactions of China Electrotechnical Society, Vol.29(S1) (China,2014), pp. 351-355. (In Chinese).

9. Niu Pingjuan. Design of High Power Factor 90W LED Street Lamp Driver. Transactions of China Electrotechnical Society, Vol.29(10) (China,2014), pp. 199-205. (In Chinese).

10. Fu Xiansong. The Design of High Power Factor LED Drive Power Supply Based on IW3623. Transactions of Electrotechnical Society, Vol.30(S1) (China,2015), pp.106-109. (In Chinese).

11. Yang Hebo. Design of drive circuit for $100 \mathrm{~W}$ wind-solar complementary LED street lamp.Modern Electronics Technique, Vol.37(4) (China,2014), pp. 159-162. (In Chinese). 
12. Yang Jianhua. Modeling and Simulation of LED Constant Current Drive Power Ship.Journal of Power Supply, 2014, 2014(4): pp.105-112. (In Chinese).

13. Chai Wandong. Remote Monitoring System Design for LED Lighting. Journal of Huaqiao University (Natural Science), Vol.37(1) (China,2016), pp. 54-57. (In Chinese).

14. Gao Luoqin. R\&D and Implementation of an embedded Remote MonitoringTerminal Based on LWIP Protocol .Electrical Automation, Vol.37(1) (China,2015), pp. 49-51. (In Chinese).

15. American Linear Technology Corporation. LT3755 manual. http://www. linear.com, 2012. 